\title{
Salvage endoscopic submucosal dissection for esophageal adenocarcinoma arising during radiofrequency ablation
}

\author{
Sarra Oumrania*, Maximilien Barret ${ }^{\mathrm{a}, \mathrm{b} *}$, Frédéric Beuvon ${ }^{\mathrm{b}, c}$, Sarah Leblanc ${ }^{\mathrm{a}}$, Stanislas Chaussade ${ }^{\mathrm{a}, \mathrm{b}}$, \\ Frédéric Prat ${ }^{\mathrm{a}, \mathrm{b}}$ \\ Cochin Hospital, Assistance Publique-Hôpitaux de Paris; Paris-Descartes University; Cochin Hospital, Paris, France \\ *These authors contributed equally to the manuscript
}

\begin{abstract}
Radiofrequency ablation is a recommended treatment option for residual Barrett's esophagus after endoscopic resection of a visible lesion. We herein report 3 cases of esophageal adenocarcinoma arising during the course of radiofrequency ablation, all of which were successfully resected by endoscopic submucosal dissection. Partial or suboptimal response to radiofrequency ablation or early recurrence of Barrett's mucosa after radiofrequency ablation should raise suspicion for adenocarcinoma and lead to consideration of en bloc resection by endoscopic submucosal dissection.
\end{abstract}

Keywords Barrett's esophagus, adenocarcinoma, radiofrequency, endoscopic submucosal dissection

Ann Gastroenterol 2018; 31 (4): 1-3

\section{Introduction}

Barrett's esophagus (BE) is defined by the replacement of the normal squamous epithelium of the tubular esophagus by intestinal metaplasia [1]. The risk of progression of $\mathrm{BE}$ to adenocarcinoma depends on the presence of dysplasia, ranging from $0.2-0.5 \%$ per year for non-dysplastic BE to $7 \%$ per year for patients with high-grade dysplasia [1]. A two-step treatment paradigm, starting with the endoscopic resection of any visible abnormality on the Barrett's segment, followed by the ablation of the residual $\mathrm{BE}$ with several sessions of radiofrequency ablation (RFA), has emerged as the standard of care for dysplastic BE $[1,2]$. This treatment protocol, typically spanning over 6-9 months, requires 2-3 RFA sessions and yielded complete remission of dysplasia and complete remission of intestinal metaplasia in $92 \%$ and $87 \%$ of cases, respectively, at two years

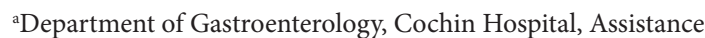
Publique-Hôpitaux de Paris (Sarra Oumrani, Maximilien Barret, Sarah Leblanc, Stanislas Chaussade, Frédéric Prat); 'Paris-Descartes University (Maximilien Barret, Frédéric Beuvon, Stanislas Chaussade, Frédéric Prat); 'Department of Pathology, Cochin Hospital (Frédéric Beuvon), Paris, France

Conflict of Interest: None

Correspondence to: Maximilien Barret, MD, PhD, Department of Gastroenterology, Cochin Hospital, 27 rue du Faubourg St Jacques 75014 Paris, France, e-mail: maximilien.barret@aphp.fr

Received 11 January 2018; accepted 26 February 2018; published online 27 April 2018

DOI: https://doi.org/10.20524/aog.2018.0261 in a recent multicenter international trial [2]. We report 3 cases of neoplastic progression occurring during treatment by RFA.

\section{Case 1}

The first case was a 71-year-old male patient with a recently diagnosed C5M6 BE, who underwent endoscopic submucosal dissection (ESD) of a $4 \mathrm{~cm}$ Barrett's segment for a nodular, Paris 0-IIa, $15 \mathrm{~mm}$ large lesion. Histopathology showed a pT1a/m3 adenocarcinoma with R0 curative resection (lateral and deep margins free of cancer and absence of poor histoprognostic factor), but with the presence of high-grade dysplasia on one lateral margin. At the 3-month follow-up endoscopy, multifocal high-grade dysplasia was found on the biopsies of the $\mathrm{C} 1 \mathrm{M} 3$ residual BE (Fig. 1). The patient underwent one RFA session (HALO 360) 6 months after endoscopic resection. Two months later, a suspicious nodule was found in the residual BE (Fig. 2A,B) and was resected en bloc by ESD. Pathology revealed a well differentiated intramucosal $\mathrm{T} 1 \mathrm{a} / \mathrm{m} 3$ adenocarcinoma with histologically complete (R0) resection and all lateral margins in squamous mucosa (Fig. 2C,D).

\section{Case 2}

A 50-year-old male patient, previously treated over two years by repeated endoscopic mucosal resection (EMR) for a C5M10 $\mathrm{BE}$ with high-grade dysplasia, subsequently underwent a total of 
4 RFA sessions at 2-month intervals. High-definition endoscopy and biopsies did not reveal any lesion suspicious for carcinoma, either before or during RFA. At the 3-month follow-up endoscopy after the fourth RFA session, a residual C2M4 nodular BE was found (Fig. 3A,B,C). Therefore, wide resection by ESD of the nodular part and most of the residual Barrett's segment was performed, including two thirds of the esophageal circumference over $6 \mathrm{~cm}$ in length. Pathology revealed a $1.2 \mathrm{~cm}$ well differentiated intramucosal $\mathrm{T} 1 \mathrm{a} / \mathrm{m} 2$ adenocarcinoma, partially covered by neosquamous mucosa with histologically complete (R0) and curative resection. At 6-month follow up, a C0M1 BE was seen with non-dysplastic intestinal metaplasia on biopsies, unchanged at 12 months under treatment with a proton pump inhibitor.

\section{Case 3}

A 76-year-old male patient underwent EMR of a visible lesion bearing high-grade dysplasia arising on a C2M10
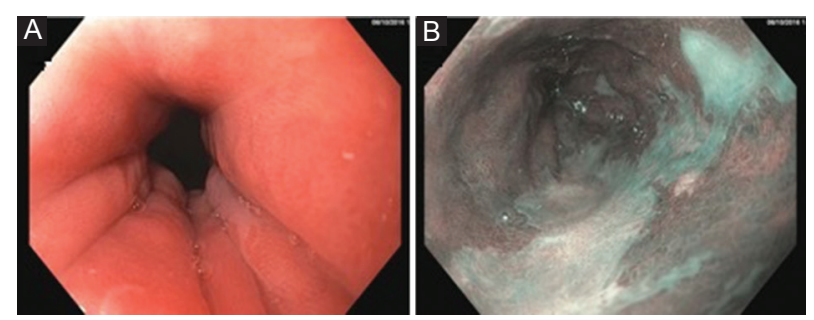

Figure 1 Case 1: Residual Barrett's esophagus in high-grade dysplasia after resection of an intramucosal carcinoma. (A) White-light endoscopy; (B) virtual chromoendoscopy by narrow-band imaging. The endoscopic resection scar is partially covered by neosquamous epithelium, from 3 to 7 o'clock

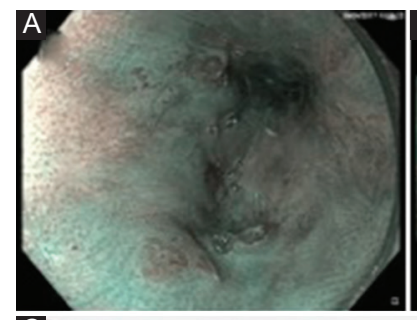

C

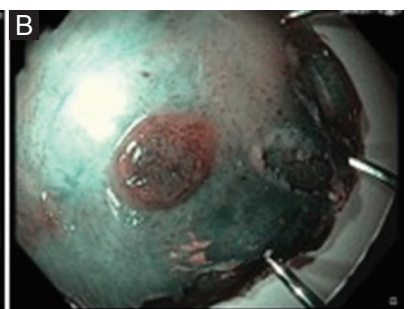

D

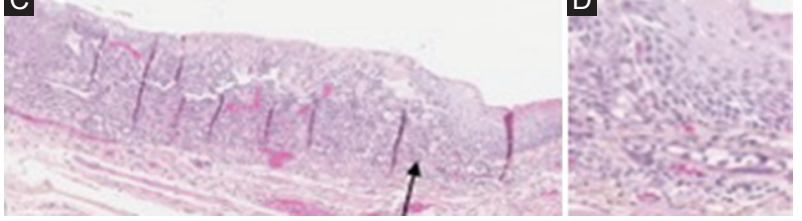

Figure 2 Case 1. (A) Virtual chromoendoscopy by narrow-band imaging, showing residual Barrett's esophagus after 1 radiofrequency ablation sessions, with suspicious nodular lesions at positions from 6 to 12 o'clock, partially covered with neosquamous epithelium. (B) Endoscopic picture under narrow-band imaging of the resected specimen. (C, D) Pathology slides (hematoxylin and eosin) showing the adenocarcinoma partially covered by neosquamous epithelium (arrow on panel C), at low- (panel C) and high- (panel D) power magnification
BE. High-definition endoscopy and biopsies of the residual C2M9 BE found no suspicious residual lesion, and the patient was scheduled for RFA eradication of the residual BE. After one RFA treatment session (Fig. 4A), the second session scheduled 2 months later was canceled, because two suspicious nodules had appeared on the residual $\mathrm{BE}$ (Fig. 4B,C). Adenocarcinoma was found on biopsies. An ESD was performed to remove two thirds of the esophageal circumference and the complete length of the Barrett's segment. Pathology showed well differentiated intramucosal $\mathrm{T} 1 \mathrm{a} / \mathrm{m} 3$ adenocarcinoma with histologically complete resection and no lymphovascular involvement (R0 curative resection). Follow-up was discontinued when the patient died from a primary pulmonary adenocarcinoma 11 months later.

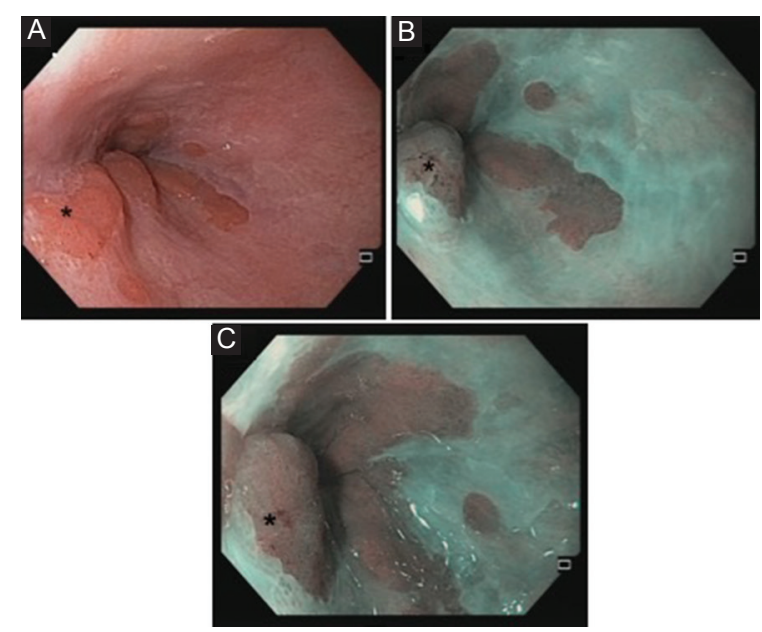

Figure 3 Case 2. White-light (A) and narrow-band imaging (B, C) showing nodular Barrett's esophagus with in situ adenocarcinoma at 8 o'clock $\left(^{*}\right) 2$ months after four radiofrequency ablation sessions

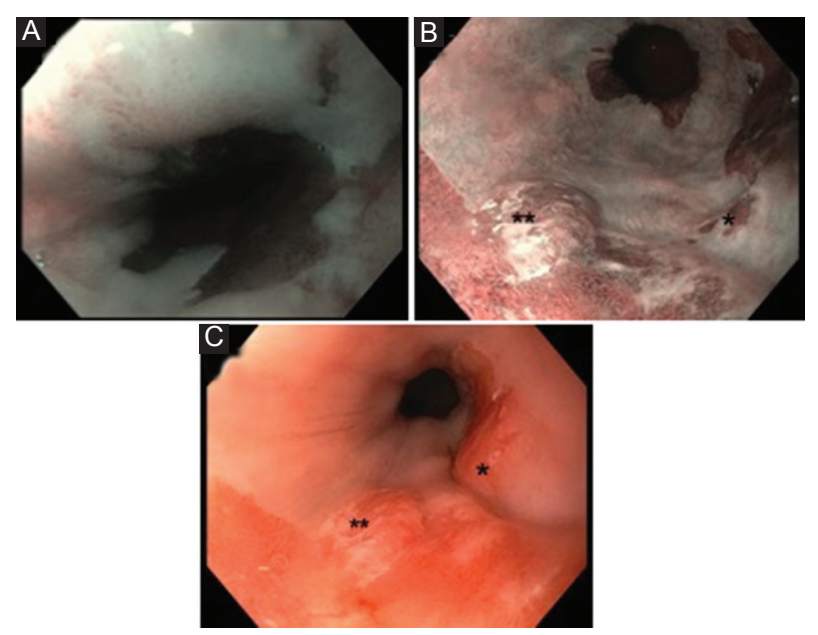

Figure 4 Case 3. Endoscopic images under narrow-band imaging showing C2M9 Barrett's esophagus before radiofrequency ablation (A) and an adenocarcinoma developing during treatment under white-light imaging (B) and narrow-band imaging (C), with 2 nodular areas at $5\left(^{*}\right)$ and $7\left({ }^{*}\right)$ o'clock 


\section{Discussion}

This report describes 3 patients who developed early adenocarcinoma during or shortly after completing RFA therapy and were ultimately cured by rescue endoscopic submucosal dissection. Leaving in place residual Barrett's mucosa after endoscopic resection of a visible, neoplastic lesion leads to the occurrence of metachronous neoplasia in up to $21.5 \%$ of patients at 5 years [3]. Indeed, surgical series have reported the presence of multifocal neoplastic foci in up to $30 \%$ of patients operated for an early Barrett's cancer [4], some of which might be overlooked by the endoscopist. For these reasons, and given its good efficacy and safety profile, RFA is the recommended therapeutic modality to eradicate residual $\mathrm{BE}$ after endoscopic resection [1].

After a median of 3 ablation sessions, RFA allows for complete eradication of intestinal metaplasia and neoplasia in up to $90 \%$ and $94 \%$ of patients at 5 years, respectively [2]. Progression to adenocarcinoma during treatment has been reported to occur in $0.8-1.5 \%$ [2,5]. Haidry et al reported salvage endoscopic resection using RFA for visible lesions arising during RFA treatment in 5-6\% of patients, but did not provide details on the lesions resected and the outcomes of this specific patient group [6]. Given the likelihood of invasive carcinoma, the pretreated esophageal mucosa with more difficult submucosal lifting or lesion suctioning, and the advantages of a large en bloc resection of the residual BE, we consider ESD should be preferred over EMR: all the more recent prospective studies have demonstrated the safety of ESD for Barrett's neoplasia, but also its superiority over EMR in terms of complete remission of dysplasia [7-9]. Although the current guidelines of the European Society of Gastrointestinal Endoscopy, released in 2015 [10], still favor EMR in most cases, these recent results should prompt an increase in the use of ESD in the treatment of early Barrett's neoplasia in treatmentnaïve and in pretreated patients in the near future.

The risk of neoplastic progression or recurrence during RFA treatment is low, but justifies a meticulous endoscopic follow up before proceeding with further ablation sessions. When a visible lesion is detected, we suggest RFA should be withheld and en bloc ESD be considered a priority.

\section{References}

1. Shaheen NJ, Falk GW, Iyer PG, Gerson LB; American College of Gastroenterology. ACG Clinical Guideline: Diagnosis and management of Barrett's esophagus. Am J Gastroenterol 2016;111:30-50.

2. Phoa KN, Pouw RE, Bisschops R, et al. Multimodality endoscopic eradication for neoplastic Barrett oesophagus: results of an European multicentre study (EURO-II). Gut 2016;65:555-562.

3. Pech O, Behrens A, May A, et al. Long-term results and risk factor analysis for recurrence after curative endoscopic therapy in 349 patients with high-grade intraepithelial neoplasia and mucosal adenocarcinoma in Barrett's oesophagus. Gut 2008;57:1200-1206.

4. Altorki NK, Lee PC, Liss Y, et al. Multifocal neoplasia and nodal metastases in T1 esophageal carcinoma: implications for endoscopic treatment. Ann Surg 2008;247:434-439.

5. Shaheen NJ, Sharma P, Overholt BF, et al. Radiofrequency ablation in Barrett's esophagus with dysplasia. $N$ Engl J Med 2009;360:2277-2288.

6. Haidry RJ, Lipman G, Banks MR, et al. Comparing outcome of radiofrequency ablation in Barrett's with high grade dysplasia and intramucosal carcinoma: a prospective multicenter UK registry. Endoscopy 2015;47:980-987.

7. Barret M, Cao DT, Beuvon F, et al. Endoscopic submucosal dissection for early Barrett's neoplasia. United European Gastroenterol J 2016;4:207-215.

8. Subramaniam S, Chedgy F, Longcroft-Wheaton G, et al. Complex early Barrett's neoplasia at 3 Western centers: European Barrett's Endoscopic Submucosal Dissection Trial (E-BEST). Gastrointest Endosc 2017;86:608-618.

9. Subramaniam S, Kandiah K, Chedgy F, Meredith P, LongcroftWheaton G, Bhandari P. The safety and efficacy of radiofrequency ablation following endoscopic submucosal dissection for Barrett's neoplasia. Dis Esophagus 2018;31.

10. Pimentel-Nunes P, Dinis-Ribeiro M, Ponchon T, et al. Endoscopic submucosal dissection: European Society of Gastrointestinal Endoscopy (ESGE) Guideline. Endoscopy 2015;47:829-854. 